\title{
Geleitwort von ao. Univ.-Prof. Dr. Herwig Ostermann
}

Über weite Strecken wird im Medizinstudium ein individualmedizinischer Blick auf Gesundheitsrisiken und -probleme eingenommen. Public Health erweitert dieses Blickfeld um populationsbezogene Sichtweisen und gesellschaftliche Einflussfaktoren. Dieses Feld entwickelt sich stetig, zuletzt sind Globalisierung und Klimawandel, soziale Ungleichheit, die demografisch bedingten Änderungen im Krankheitsspektrum zunehmend in den Fokus dieser multidisziplinären Wissenschaft gerückt. In der Theorie analysiert Public Health epidemiologisch fassbare Risikostrukturen, Verursachungszusammenhänge und Bewältigungsstrategien. In der (politischen) Praxis sollten diese Erkenntnisse in allen gesundheitsrelevanten Handlungsfeldern Berücksichtigung finden. Kaum jemand, der im Gesundheitswesen tätig ist, kann sich dieser Dimension entziehen.

Gerade in Zeiten einer Pandemie haben wir zuletzt gesehen, dass ein breiter und umfassender Blick auf das Geschehen notwendig ist. Neben der Rettung einzelner Menschenleben geht es auch um Maßnahmen zur Eindämmung der Ausbreitung der Erkrankung und zum Schutz des Lebens von Vielen. Dabei rücken zwangsläufig auch Fragen der Angemessenheit von Maßnahmen sowie ihrer Folgen für die Gesellschaft in den Fokus. Es gilt, Kollateralschäden wie weitere Verschärfungen der sozialen und gesundheitlichen Ungleichheit sowie physische und psychische Langfristschäden zu verhindern. Public Health übernimmt hier eine wichtige Sichtweise und Aufgabe. Dazu braucht es neben der Medizin eine Vielzahl von Disziplinen und Methoden - u. a. Epidemiologie, Gesundheitsökonomie, Gesundheitssystem- und Versorgungsforschung, Prävention und Gesundheitsförderung, Soziologie, Psychologie, Gesundheitskommunikation sowie Ethik.

Das Lehrbuch Public Health kompakt, nun in der 4. Auflage erschienen, gibt einen ausgezeichneten Überblick über die Konzepte und Handlungsfelder von Public Health und einen spannenden Einstieg in die Welt der Bevölkerungsgesundheit.

ao. Univ.-Prof. Dr. Herwig Ostermann

Geschäftsführer Gesundheit Österreich GmbH, Wien 
